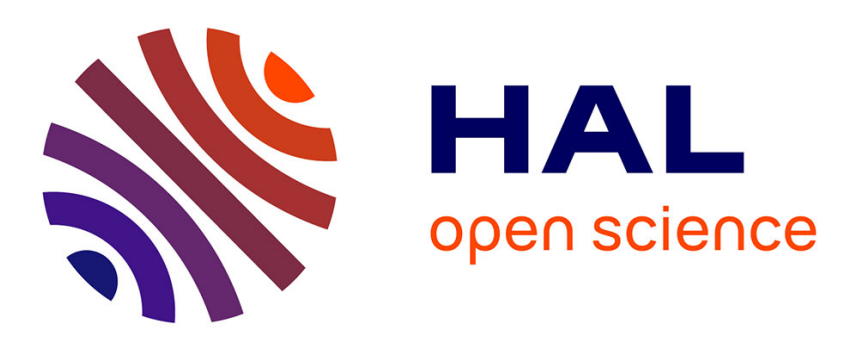

\title{
Electrical and optical measurements of the bandgap energy of a light-emitting diode
}

Matthieu Petit, Lisa Michez, Jean-Manuel Raimundo, Philippe Dumas

\section{To cite this version:}

Matthieu Petit, Lisa Michez, Jean-Manuel Raimundo, Philippe Dumas. Electrical and optical measurements of the bandgap energy of a light-emitting diode. Physics Education, 2016, 51 (2), pp.025003. 10.1088/0031-9120/51/2/025003 . hal-01266699

\section{HAL Id: hal-01266699 \\ https://hal-amu.archives-ouvertes.fr/hal-01266699}

Submitted on 3 Feb 2016

HAL is a multi-disciplinary open access archive for the deposit and dissemination of scientific research documents, whether they are published or not. The documents may come from teaching and research institutions in France or abroad, or from public or private research centers.
L'archive ouverte pluridisciplinaire HAL, est destinée au dépôt et à la diffusion de documents scientifiques de niveau recherche, publiés ou non, émanant des établissements d'enseignement et de recherche français ou étrangers, des laboratoires publics ou privés. 


\title{
Electrical and optical measurements of the bandgap energy of a light-emitting diode.
}

\author{
Matthieu Petit, Lisa Michez, Jean-Manuel Raimundo, \\ Philippe Dumas \\ Aix-Marseille Université, CNRS, CINaM UMR 7325, 13288, Marseille, France. \\ E-mail: matthieu.petit@univ-amu.fr
}

\begin{abstract}
Semiconductors materials are at the basis of electronics. Most electronic devices are made of semiconductors. The operation of these components is well described by quantum physics which is a little mysterious to students. One of the intrinsic parameters of semiconductors is their bandgap energy $E_{g}$. In the case of light-emitting diodes (LEDs) $E_{g}$ fixes the colour of the light emitted by the diodes. We propose in this article a lab work allowing a comparison of $E_{g}$ of a green LED obtained by both electrical and optical measurements. The two slightly different results can be explained by the theoritical knowledges of the students on solid physics and electronic devices internal architecture.
\end{abstract}

PACS numbers: 71.20.Nr, 73.40.Lq, 73.40.-c

Keywords: bandgap, semiconductors, p-n junctions, LED, light-emitting diode 


\section{Introduction}

\subsection{Context}

Semiconductors materials are at the basis of electronics [1]. Most of the electronic devices are made of semiconductors. The functioning of semiconductors devices is completely described by quantum physics in the band theory of electrons in solids. The concept of band structure in the physics of semiconductors often retains an element of mystery for our students of first year of school of engineering (third year of university class) specialized in materials science. Indeed the notions developed in this theory are somewhat abstract. Therefore it is interesting to provide students with simple experiments in order to manipulate these notions. A description of the solid physics of semiconductors materials and of the physics of the semiconductors devices can be found in the books of C Kittel [2] and S M Sze [3]. The band structure of a crystalline solid is the relationship between the energy $\mathrm{E}$ of the electrons of the solid and the wave vector $k$ (i.e. momentum). It comes from the Schrödinger equation (eq.1) of an approximate oneelectron problem in a periodic potential $U(\boldsymbol{r})$ with the periodicity of the crystal lattice:

$$
\left(-\frac{\hbar^{2}}{2 m} \nabla^{2}+U(\boldsymbol{r})\right) \psi_{k}(\boldsymbol{r})=E_{k} \psi_{k}(\boldsymbol{r})
$$

(r) is a vector of the direct lattice, $\psi_{k}(\boldsymbol{r})$ the wave function describing the given electron, $E_{k}$ and $\mathrm{m}$ its energy and mass respectively. In the case of a periodic crystal, the Bloch's theorem gives the form of the solutions of the Schrödinger equation. There are energy $E_{k}$ domains where no solution exist. These domains are forbidden: electrons cannot have energies with values belonging to these regions. Above and below this energy gap are permitted energy regions called bands. The upper band is the conduction band $(\mathrm{CB})$, the lower band is the valence band $(\mathrm{VB})$ as displayed on fig.1a). If we called $E_{C B}$ the smallest energy of the conduction band and $E_{V B}$ the highest energy of the valence band, the forbidden energy gap range $E_{g}$ is equal to (eq.2):

$E_{g}=E_{C B}-E_{V B}$

$E_{g}$ is the bandgap energy, one the most important parameters in semiconductor physics. Each semiconductor material has a specific bandgap. For instance silicon ( $\mathrm{Si}$ ) has a bandgap value of 1.12 electronvolt $(\mathrm{eV})$ and germanium $0.66 \mathrm{eV}$ at room temperature (RT).

External excitations such as temperature, light, electric current can enable the "jump" of an electron from $\mathrm{VB}$ to the $\mathrm{CB}$. The energy of this electron increases by a value equal to $E_{g}$. The "jumping"

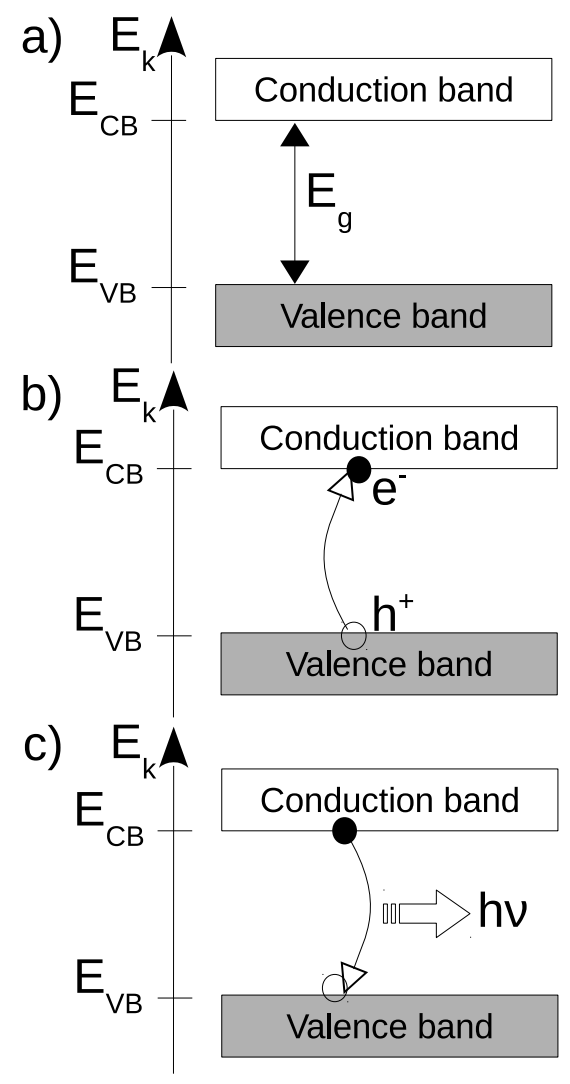

Figure 1. a) Simplified band diagram of a semiconductor, b) in an excited state with an electron-hole pair, c) when a radiative transition occurs emitting a photon with an energy $E_{g}=h \nu$.

electron leaves a positive charge called "hole" in the valence band. The excitation creates an electron-hole pair (fig.1b)). The electron can lose energy and goes down to the VB. The lost energy might be transferred to a photon (radiativ transition) and is also equal to $E_{g}$ as shown in fig.1c). This phenomenon is the luminescence and is used in optoelectronics devices like light-emitting diode (LED).

\subsection{Electrical approach for the lab work}

Students measure the bandgap of semiconductor materials used in commercially available LEDs. In the case of the lab work and to simplify the problem on an electrical point of view, these devices are described as simple p-n junctions. To get a good understanding of the functioning of a p-n junction, we suggest the reader to read the article of $\mathrm{E}$. Boyes [4]. The electrical current I through a p-n junction is given by:

$I=I_{\text {sat }}\left(e^{\frac{q V}{k^{T}}}-1\right)$

$\mathrm{V}$ is the voltage applied to the p-n junction, $\mathrm{q}$ the elementary charge, $k_{B}$ the Boltzmann constant and $\mathrm{T}$ the temperature. The current-voltage characteristic of an ideal p-n junction is drawn on fig. 2 . 


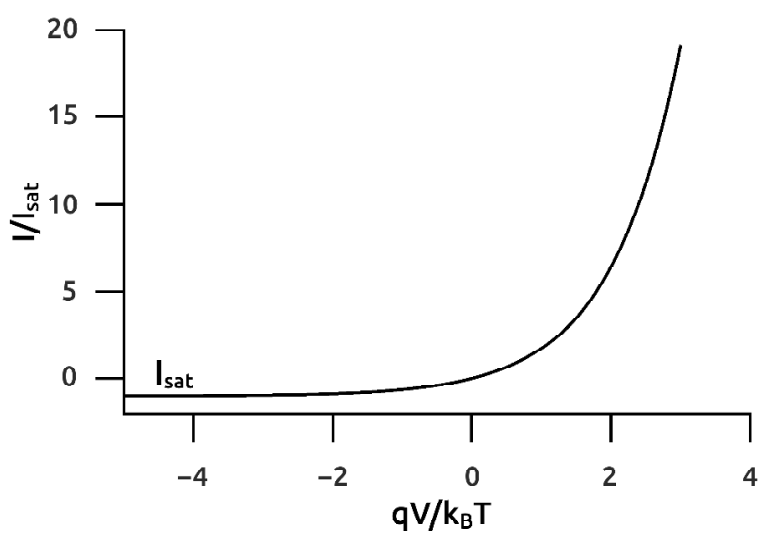

Figure 2. Ideal current-voltage characteristic of a p-n junction.

$I_{\text {sat }}$ is a function of $\mathrm{T}$ and of the bandgap [3]. It can be written as:

$I_{\text {sat }}=T^{3+\frac{\gamma}{2}} e^{\left(-\frac{E_{g}}{k_{B} T}\right)}$

$\gamma$ is a constant depending on a given semiconductor material with a value in the range of 1 to 2 . Thus the total expression of the current is:

$I=T^{3+\frac{\gamma}{2}}\left(e^{-\frac{E g}{k_{B} T}}\right)\left(e^{\frac{q V}{k_{B} T}}-1\right)$

\section{Method and implementation}

\subsection{Equipment}

The needed equipments consist firstly in a set of LEDs emitting in the visible light range. LEDs will be generally made of III-V semiconductors. III-V semiconductors are alloys from atoms of the third and fifth columns of the periodic table. They are commonly used in the optoelectronics devices. These devices are powered by a power supply in the range of $0-5 \mathrm{~V}$. A current meter with a resolution of 0.01 $\mu \mathrm{A}$ (measurement uncertainty: $\pm 0.05 \%+3$ digits) and a voltmeter with a resolution of 0.0001 volt (measurement uncertainty: $\pm 0.02 \%+2$ digits) allow to do the measure of the electrical current and applied voltage of the semiconductor devices (fig.3). A potentiometer with a range from 0 to $10 \mathrm{k} \Omega$ completes the electrical apparatus require for the lab work. Indeed there are three parameters which can varies in this experimental set-up according to eq.5: the electrical current I, the applied voltage $\mathrm{V}$ and the temperature of the electronic device T. To simplify the problem, we choose to fix either the current or the voltage with the variable resistor: adjusting the potentiometer will allow to keep the current or voltage constant. In the following parts, we will work with a constant electrical current. Finally an oven heating up to $100^{\circ} \mathrm{C}$ with a temperature regulation and a

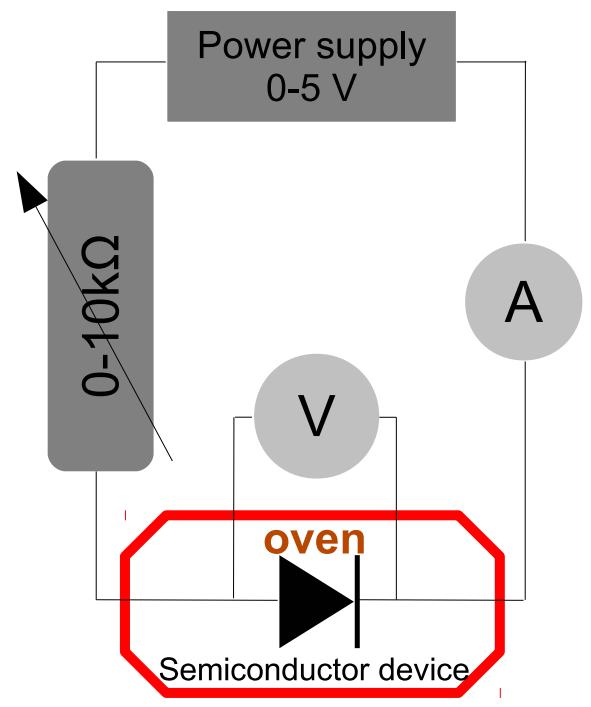

Figure 3. Electrical scheme of the experimental set-up.

step control of $1^{\circ} \mathrm{C}$ will allow to change the device temperature.

\subsection{Modelling}

In order to calculate $E_{g}$ from the electrical measurements versus temperature, eq.5 must be re-written in a simpler way taking into account the available range of the experimental parameters of the lab work: the applied voltage of the semiconductor device is around $5 \mathrm{~V}$, the temperature is in the range of $\left[R T ; 100^{\circ} \mathrm{C}\right]$. This modelling part is interesting since students have to adapt their theoretical knowledges about the p-n junction to the specific framework of this pratical work. The logarithm of eq.5 gives the following expression:

$\ln (I)=\left(3+\frac{\gamma}{2}\right) \ln (T)-\frac{E_{g}}{k_{B} T}+\ln \left(e^{\frac{q V}{k_{B} T}}-1\right)$

Taking into account the range of the experimental parameters, we can estimate that:

$e^{\frac{q V}{k_{B} T}}-1 \approx e^{\frac{q V}{k_{B} T}}$
$\left(3+\frac{\gamma}{2}\right) \ln (T) \approx$ constant
$\ln (I)=$ constant.

Using eq.7, 8 and 9 in eq.6, we get a linear relationship between the applied voltage and the temperature of the device:

$V=\frac{C}{q} T+\frac{E_{g}}{q}$

where $\mathrm{C}$ is a constant depending on the chosen fixed value of the current. Thus it is possible to estimate the value of $E_{g}$ by plotting the straight line $\mathrm{V}$ versus $\mathrm{T}$ (in Kelvin). At $\mathrm{T}=0 \mathrm{~K}, V=\frac{E_{g}}{q}$ is the value of $E_{g}$ directly in $\mathrm{eV}$. 


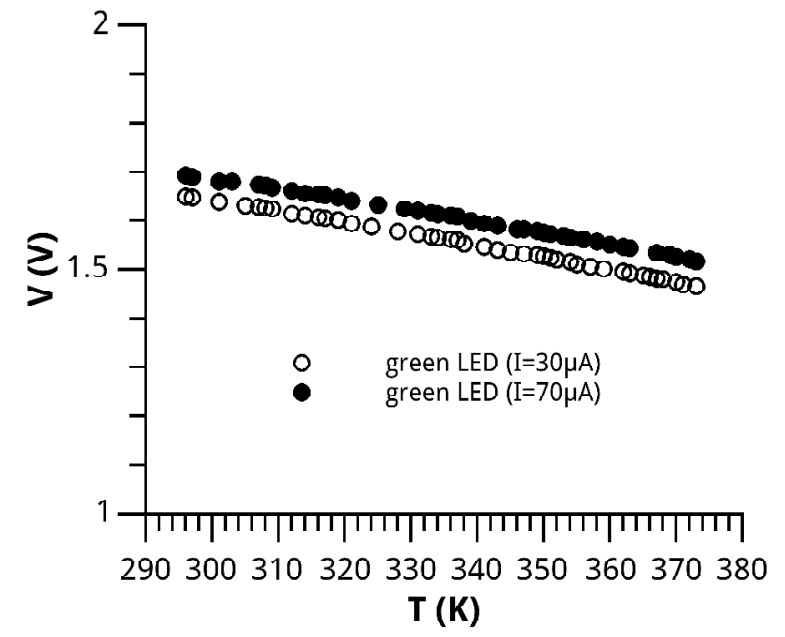

Figure 4. Evolution of the applied voltage $\mathrm{V}$ versus temperature $\mathrm{T}$ for a green LED at two different fixed electrical currents I $(30$ and $70 \mu \mathrm{A})$

\subsection{Electrical and optical measurements}

To start the electrical measurements, the students have to choose a working value for the electrical current according to the semiconductor device they study. Then the oven is programmed with a temperature ramp. The ramp should be quite low to allow a good thermalization of the electronic component and enough time to take the measures. In fig. 4 we display the measurements coming from four experiments: we have studied a green LED at two different fixed currents (30 and $70 \mu \mathrm{A}$ ). The applied voltages versus the temperature are drawn. As forecast by eq.10, we obtain linear relationships between $\mathrm{V}$ and T. Regarding the optical measurements, the light emitted spectrum of the LED is recorded with a USB-650 Red-Tide spectrometer from Ocean Optics [5]. This small spectrometer is connected via an USB port to a computer and is very user friendly.

\section{Results and discussion}

\subsection{Electrical measurements versus temperature}

The results of the linear fits of our experimental data (fig.4) are summarized in table 1.

First of all to discuss these results, students should seek what are the semiconductor materials commonly used to manufacture the LED components. Obvioulsy the reference numbers leading to the corresponding device datasheets are relevant to find informations. One of the traditional III-V semiconductor material used to produced green wavelengths is the gallium phosphide (GaP) which has a theoretical bangap energy of $2.26 \mathrm{eV}$ at $300 \mathrm{~K}$. The datasheet of our used LEDs (model L-1503GD from Kingbright) confirmes
Table 1. Results coming from the linear fits of the experimental data (fig.4)

\begin{tabular}{lcc}
\hline & \multicolumn{2}{c}{ green LED } \\
& $30 \mu \mathrm{A}$ & $70 \mu \mathrm{A}$ \\
\hline$A=\frac{C}{q}\left(\times 10^{-3}\right)$ & -2.41 & -2.28 \\
$\pm 0.02 \times 10^{-3}$ & & \\
$B=\frac{E g}{q}(\mathrm{eV})$ & 2.37 & 2.37 \\
\pm 0.06 & & \\
$R^{2}$ & 0.998 & 0.998 \\
\hline
\end{tabular}

Table 2. Values of the parameters of eq.11 [7], [8]

\begin{tabular}{llll}
\hline Semiconductor & $E_{0}(\mathrm{eV})$ & $\alpha \times 10^{-4}$ & $\beta$ \\
\hline $\mathrm{GaP}$ & 2.34 & 6 & 460 \\
\hline
\end{tabular}

the nature of the semiconductor [6]. The difference between our experimental values of $E_{g}(G a P)$ and the theoretical one are $5 \%$. In the next step of the interpretation, the students should provide some explanations to these disparities. Two types of disparities can be contemplated based on experimental and theoretical accounts. On the experimental point of view, these differences may mainly arise from the temperature measurement: we use the temperature values providing by the oven controller. But these temperatures are overestimated compare to the temperatures of the studied electronic components because of the thermal inertia of the experimental set-up. The influence of this source of errors could be decreased by setting a lower temperature ramp to ensure a better thermalization of the components but the duration of the lab work is limited. On the theoretical point of view the bandgap energy is itself a function of the temperature. This point is not taken into account in our simple electrical model of the $\mathrm{p}-\mathrm{n}$ junction. Through the Bloch's theorem, $E_{g}$ depends on the lattice parameters of the semiconductors which depend also on the temperature. Varshni propose the following relationship between $E_{g}$ and $\mathrm{T}$ [7]:

$E_{g}(T)=E_{0}-\frac{\alpha T^{2}}{T+\beta}$

where $\alpha$ and $\beta$ are constants for a given semiconductors materials. $E_{0}$ is the bandgap energy at $0 \mathrm{~K} . E_{0}, \alpha$ and $\beta$ are given in table 2 for GaP.

Thus in the temperature range of this practical work, the variation of $E_{g}$ is presented on fig.5.

The difference of $E_{g}$ between RT and $373 \mathrm{~K}$ $\left(100^{\circ} \mathrm{C}\right)$ is around $3 \%$. So this effect may be taken into account to explain the discrepancies between the value of $E_{g}$ found by our electrical measurements versus temperature and the theoretical values given at RT. 


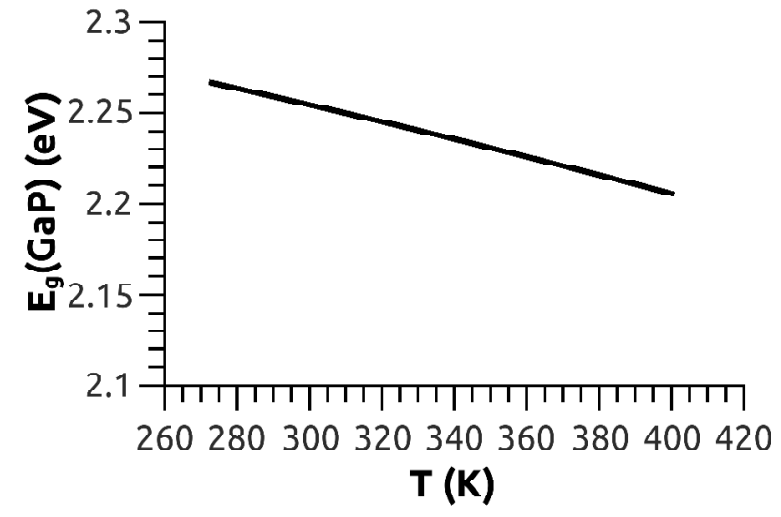

Figure 5. Variation of the gap energy with temperature for GaP according to eq.11 and table 2 .

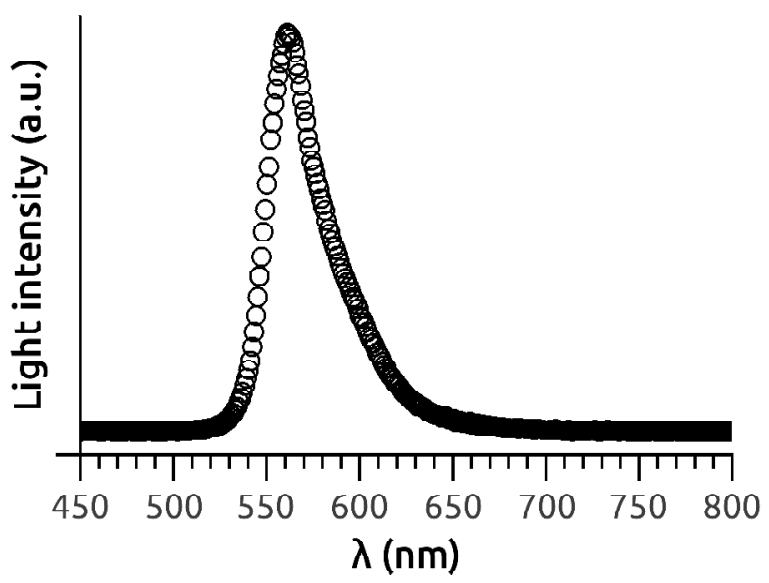

Figure 6. Light spectrum of the green LED recorded with a UV-visible spectrometer.

\section{2. optical spectrum of the green $L E D$}

In the case of the LEDs it is possible to access the (optical) bandgap energy by recording the light spectrum emitted by the device. The spectrum of the used green LED is shown on fig.6.

The maximum light intensity is recorded for a wavelength $\lambda=(561 \pm 5) \mathrm{nm}$. This corresponds to an energy of $E=\frac{h c}{\lambda}=(2.21 \pm 0.02) \mathrm{eV}$ which is suitable for a GaP based LED. The reference value of the wavelength given by the manufacturer datasheet is $\lambda_{\text {ref }}=565 \mathrm{~nm}$ corresponding to an energy of $2.20 \mathrm{eV}$.

\subsection{How to explain the difference between the electrical and optical methods?}

The last step of this lab work is to propose an explanation of the difference between electrical and optical values of $E_{g}$. The main reason is linked to how a LED is really designed. A LED is basically a p-n junction working with a forward bias. Electrons are the majority carriers in the n-type region. They

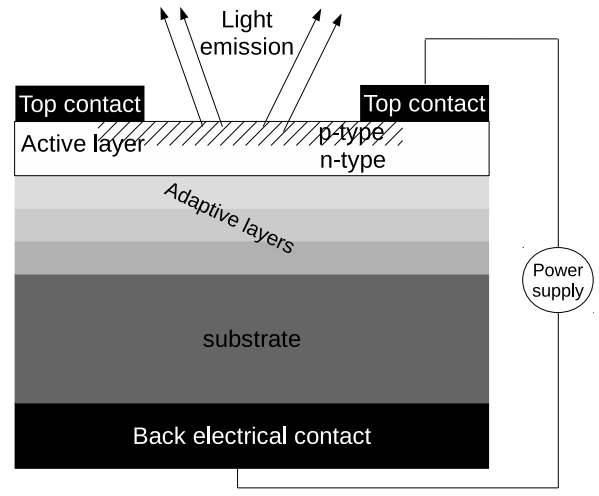

Figure 7. scheme of a LED with the electrical contacts in black, the active p-n junction layer and the successive epitaxial layers which guarantee a very good chemical and crystallographic quality of the active layer.

are injected in the p-type region where electrons can recombined with the holes. Thus LEDs are made of semiconductors where the radiative recombinations probability is high. The light colour emitted by the diode depends directly on the bandgap energy of the semiconductor materials where the radiative transition takes place. In order to get a high light emission yield a very good chemical and crystallographic quality of the materials is required. Practically it means that a LED will be built from successive epitaxial (a definition of "epitaxy" can be found on Wikipedia [9]) stacking layers of semiconductors like shown on fig.7.

Therefore the LED is not strictly speaking a simple p-n junction that we have used to model the devices for the electrical measurements. The optical measure gives the bandgap energy of the active light emitting layer alone. On the contrary the electrical experiment give access to an "average" bandgap energy of all stacking layers.

\section{Summary}

This article provides a quick description of the underlying quantum physics explaining the semiconductor devices. Basic devices such as LEDs can be approximated by p-n junctions. Thus the electrical current flowing through the component depends only on the applied voltage, the temperature and the intrinsic semiconductor bandgap energy $E_{g}$. A simple electrical set-up combined with an oven allowing the variation of the temperature gives rather good estimation of $E_{g}$. Moreover the bangap energy of LEDs can also be measure from the wavelength of the emitted light. In this lab work designed for first years university students with a major in materials science and a duration of 3 to 4 hours, we show that by this two methods the $E_{g}$ of $\mathrm{GaP}$ used in a green LED is obtained with about a $5 \%$ error. The most interesting part is for the students 
to try to explain theirs results coming from electrical and optical measurements by the various knowledges they have on solid physics and those they find doing some bibliographical researches on the internal design of optoelectronics devices.

\section{References}

[1] Jenkins T 2005 Physics Education 40430

[2] Kittel C 1996 Introduction to Solid State Physics john wiley ed (New York) ISBN 978-0471111818

[3] Sze S 1969 Physics of semiconductor devices wiley ed (New York: John Wiley and Sons Ltd.) ISBN 0-471-84290-7

[4] Boyes E 1990 Physics Education 2553

[5] Ocean Optics I [Online; accessed 04-April-2015] URL http: //oceanoptics.com/

[6] Kingbright Electronic Co L [Online; accessed 17-July-2015] URL http://www.kingbright.com

[7] Varshni Y 1967 Physica 34 149-154 ISSN 00318914

[8] Institut I [Online; accessed 04-April-2015] URL http://www . ioffe.rssi.ru/SVA/NSM/Semicond/index.html

[9] Wikipedia 2015 Epitaxy — wikipedia, the free encyclopedia [Online; accessed 17-July-2015] URL https://en.wikipedia.org/w/index.php?title= Epitaxy\&oldid=666329643 